\title{
Manding Language
}

National Cancer Institute

\section{Source}

National Cancer Institute. Manding Language. NCI Thesaurus. Code C161851.

Dialects of the Mande language family spoken in West Africa. 